\title{
COCYCLE CONJUGACY CLASSES OF BINARY SHIFTS
}

\author{
Geoffrey L. Price \\ United States Naval Academy
}

February 12, 2016

In memory of William B. Arveson

\begin{abstract}
We show that every binary shift on the hyperfinite $I I_{1}$ factor $R$ is cocycle conjugate to at least countably many non-conjugate binary shifts. This holds in particular for binary shifts of infinite commutant index.
\end{abstract}

\section{Preliminaries.}

Let $(a)=a_{0}, a_{1}, a_{2}, \ldots$ be a bitstream, i.e. a sequence of 0's and 1's in $G F(2)$. Let $v_{0}, v_{1}, v_{2}, \ldots$ be a sequence of self-adjoint unitary operators (which we shall call generators) that satisfy the translation-invariant commutation relations

$$
v_{i} v_{i+j}=(-1)^{a_{j}} v_{i+j} v_{i}
$$

for all non-negative integers $i$ and $j$. The commutation relations imply that $a_{0}=$ 0 . The norm closure of the set of all linear combinations of the words in the $v_{i}$ 's is isomorphic to the CAR algebra, $\mathfrak{A}$, if and only if the mirror sequence $\ldots, a_{2}, a_{1}, a_{0}, a_{1}, a_{2}, \ldots$ is not periodic, [ $\operatorname{Pr} 87$, Theorem 2.3]. Using the GNS representation for the unique trace on $\mathfrak{A},[\mathrm{KR}]$, which sends all non-trivial words in the $v_{i}$ 's to 0 , the hyperfinite $I I_{1}$ factor $R$ is obtained as the strong closure of the set of all linear combinations of the words in the $v_{i}$ 's. Then the mapping sending $v_{i}$ to $v_{i+1}$ gives rise to a unital $*$-homomorphism $\alpha$ on $R$, called a binary shift. The image $\alpha(R)$ has subfactor index 2, i.e. $[R: \alpha(R)]=2$. Note that $R$ is generated by $\alpha(R)$ and $v_{0}$. 
Definition 1, cf. [Po]. Two unital *-endomorphisms $\alpha$ and $\beta$ on $R$ are said to be conjugate if there is a $*$-automorphism $\gamma$ of $R$ such that

$$
\alpha(v)=\gamma\left(\beta\left(\gamma^{-1}(v)\right)\right), \text { for all } v \in R
$$

Two unital $*$-endomorphisms $\alpha$ and $\beta$ are said to be cocycle conjugate if there is a unitary $u \in R$ so that $A d(u) \circ \alpha$ and $\beta$ are conjugate.

Theorem 1. [Po, Theorem 3.6] Binary shifts $\alpha$ and $\beta$ on $R$ are conjugate if and only if they have the same bitstream.

For $n \in \mathbb{N}$ let $\mathcal{A}_{n}$ be the $n \times n$ Toeplitz matrix over $G F(2)$, given by

$$
\mathcal{A}_{n}=\left[\begin{array}{cccccc}
a_{0} & a_{1} & a_{2} & a_{3} & \ldots & a_{n-1} \\
a_{1} & a_{0} & a_{1} & a_{2} & \ldots & a_{n-2} \\
a_{2} & a_{1} & a_{0} & a_{1} & \ldots & a_{n-3} \\
\vdots & \vdots & \vdots & \vdots & \ddots & \vdots \\
a_{n-1} & a_{n-2} & a_{n-3} & \ldots & \ldots & a_{0}
\end{array}\right]
$$

Note that $\mathcal{A}_{n}$ is symmetric with main diagonal all 0's. For each $n \in \mathbb{N}$ let $\nu_{n}=\operatorname{null}\left(\mathcal{A}_{n}\right)$, i.e. the dimension of $\operatorname{ker}\left(\mathcal{A}_{n}\right)$. The importance of the nullity sequence $\left\{\nu_{n}: n \in \mathbb{N}\right\}$ is that it gives information about the centers of the algebras $\mathfrak{A}_{n}$ generated by $v_{0}, \ldots, v_{n-1}$ (see Theorem 3 and Theorem 4 below).

Theorem 2. [PP, Theorem 5.4], [CP, Theorem 2.7] The sequence $\left\{\nu_{n}: n \in \mathbb{N}\right\}$ is the concatenation of finite length strings of the form 1,0 or $1,2, \ldots, r-1, r, r-$ $1, \ldots, 0$ for some $r>1$, where the length $2 r$ of the string may vary.

Remark 1. If the mirror bitstream is periodic there is a non-negative integer $n$ such that $\nu_{n+j+1}=\nu_{n+j}+1$ for all $j \in \mathbb{N}$, [Pr98], and in this case the $C^{*}$-algebra $C^{*}\left(v_{0}, v_{1}, \ldots\right)$ is isomorphic to $\mathcal{T} \otimes C(X)$, where $X$ is the Cantor set, and $\mathcal{T}$ is either $\mathbb{C} I$ or the tensor product of finitely many copies of $M_{2}(\mathbb{C}),[\mathrm{AP}$, Theorem $3.1]$.

Theorem 3. [PP, Corollary 5.5],[Pr98, Lemma 3.2] Let $\alpha$ be a binary shift on $R$ with bitstream $(a)$. For any positive integer $n$ there exists a one-to-one correspondence between vectors $\mathbf{c}=\left[c_{0}, c_{1}, \ldots, c_{n-1}\right]^{t}$ in $\operatorname{ker}\left(\mathcal{A}_{n}\right)$ and ordered words $v_{0}^{c_{0}} v_{1}^{c_{1}} \cdots v_{n-1}^{c_{n-1}}$ in the center $\mathfrak{Z}_{n}$ of $\mathfrak{A}_{n}$. Therefore, as $\mathfrak{Z}_{n}$ consists of linear combinations of the words it contains, its dimension as an algebra over $\mathbb{C}$ is $2^{\nu_{n}}$. In particular, if $\nu_{n}=0$ then $\mathfrak{Z}_{n}=\mathbb{C} I$ and $\mathfrak{A}_{n}$ is a full matrix algebra over $\mathbb{C}$.

The following result about the centers $\mathfrak{Z}_{n}$ of the algebras $\mathfrak{A}_{n}$, along with the correspondence between $\mathfrak{Z}_{n}$ and $\operatorname{ker}\left(\mathcal{A}_{n}\right)$ discussed above, shows that to understand the structure of $\operatorname{ker}\left(\mathcal{A}_{n}\right)$ for all $n$ it suffices to know the structure of $\operatorname{ker}\left(\mathcal{A}_{n}\right)$, where $\nu_{n-1}=0$ and, (therefore necessarily, by Theorem 2), $\nu_{n}=1$. 
Theorem 4. [PP, Lemma 6.5],[Pr98, Theorem 3.4] Let $n \in \mathbb{N}$ and $d \in \mathbb{N}$ be such that the nullities $\nu_{n}$ through $\nu_{n+d-1}$ are 1 through $d$, respectively, and $\nu_{n+d}$ through $\nu_{n+2 d-1}$ are $d-1$ through 0 , respectively. Let $z=v_{0}^{c_{0}} v_{1}^{c_{1}} \cdots v_{n-1}^{c_{n-1}}$ be the word generating $\mathfrak{Z}_{n}$, then the exponents $c_{0}, c_{1}, \ldots, c_{n-1}$ form a palindrome, i.e. $\left[c_{0}, c_{1}, \ldots, c_{n-1}\right]=\left[c_{n-1}, c_{n-2}, \ldots, c_{0}\right]$, with $c_{0}=c_{n-1}=1$. For $m=0,1, \ldots, d-1$, the center $\mathfrak{Z}_{n+m}$ is generated by the words $z, \alpha(z), \cdots, \alpha^{m}(z) . \mathfrak{Z}_{n+d}$ is generated by $\alpha(z), \ldots, \alpha^{d-1}(z), \mathfrak{Z}_{n+d+1}$ by $\alpha^{2}(z), \ldots, \alpha^{d-1}(z)$, and so on, and $\mathfrak{Z}_{n+2 d-1}$ is trivial.

2. Main Result. We now construct countably many binary shifts that are cocycle conjugate to a given binary shift $\alpha$. Fix $n \in \mathbb{N}$ such that $\nu_{n-1}=0$ and $\nu_{n}=1$. As above, let $z=v_{0}^{c_{0}} v_{1}^{c_{1}} \cdots v_{n-1}^{c_{n-1}}$ be the word generating $\mathfrak{Z}_{n}$, the center of the algebra $\mathfrak{A}_{n}$ generated by $v_{0}, v_{1}, \ldots, v_{n-1}$. By Theorem 4 , the exponents of $z$ form a palindrome. Define a unitary operator $u$ by

$$
u=\left\{\begin{array}{l}
\frac{I+z}{\sqrt{2}}, \text { if } z^{*}=-z \\
\frac{I+i z}{\sqrt{2}}, \text { if } z^{*}=z
\end{array}\right.
$$

Note for any word $w$ in the generators,

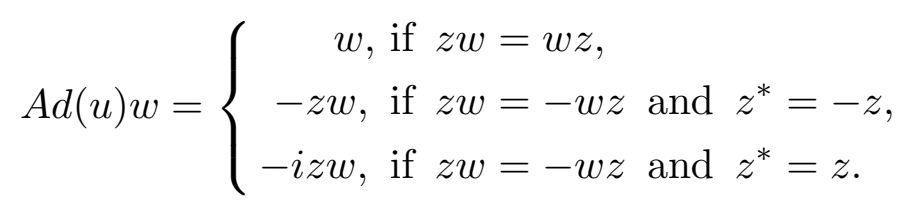

Set $\beta=A d(u) \circ \alpha, u_{0}=v_{0}$ and for $i \in \mathbb{N}$, let $u_{i}=\beta^{i}\left(v_{0}\right)$. Since $z$ commutes with $v_{0}, v_{1}, \ldots, v_{n-1}$ it follows that $u_{i}=v_{i}$ for $i=0,1, \ldots, n-1$.

First suppose $\nu_{n+1}=0$. Then $\mathfrak{Z}_{n+1}$ is trivial. Since $z \in \mathfrak{Z}_{n}$, and therefore commutes with $v_{0}$ through $v_{n-1}$, we conclude that $z$ anticommutes with $v_{n}$ (otherwise $z$ would be in $\mathfrak{Z}_{n+1}$, which is false). So we have

$$
u_{n}=\beta^{n}\left(v_{0}\right)=\beta\left(\beta^{n-1}\left(v_{0}\right)\right)=\beta\left(v_{n-1}\right)=u^{*} v_{n} u=\lambda_{0} z v_{n}
$$

(where $\lambda_{0}$ is -1 or $-i$ ), and so $u_{n+1}=\beta\left(u_{n}\right)=u^{*}\left(\lambda_{0} \alpha(z) v_{n+1}\right) u$ is either $\lambda_{1} z \alpha(z) v_{n+1}$ or $\lambda_{1} \alpha(z) v_{n+1}$ with $\lambda_{1}$ a fourth root of unity. Write $u_{n+1}$ as $\lambda_{1} z^{r_{0}} \alpha(z) v_{n+1}$. Using the symmetry arising from the fact that the exponent pattern of $z$ forms a palindrome, we conclude that since $z$ anticommutes with $v_{n}$, then $\alpha(z)$ anticommutes with $v_{0}$. Also $v_{0}$ commutes with $z$. Since $u_{0}=v_{0}$ and since $u_{n+1}=\lambda_{1} z^{r_{0}} \alpha(z) v_{n+1}$, we conclude that $b_{n+1} \neq a_{n+1}$. Therefore $\alpha$ and $\beta$ have different bitstreams.

Finally observe that the operators $u_{0}, u_{1}, \ldots, u_{n-1}, u_{n}, u_{n+1}, u_{n+2}, \ldots$ are the operators $v_{0}, v_{1}, \ldots, v_{n-1}, \lambda_{0} z v_{n}, \lambda_{1} z^{r_{0}} \alpha(z) v_{n+1}, \lambda_{2} z^{r_{1}} \alpha(z)^{r_{0}} \alpha^{2}(z) v_{n+2}, \ldots$ for some 
scalars $\lambda_{i}$ of modulus 1. Since $z \in \mathfrak{A}_{n}$ it is clear that for all $m \in \mathbb{N}, \mathfrak{B}_{m}=\mathfrak{A}_{m}$, where $\mathfrak{B}_{m}$ is the algebra generated by $u_{0}, \ldots, u_{m-1}$. So $\beta$ is a binary shift (with unitary generators $u_{0}, u_{1}, \ldots$ and bitstream $\left.(b)=b_{0}, b_{1}, \ldots\right)$ which is cocycle conjugate to $\alpha$. Since $\alpha$ and $\beta$ have different bitstreams they are not conjugate, by Theorem 1.

Next suppose the nullity sequence for $\alpha$ satisfies the hypotheses of Theorem 4 with $d \geq 2$. Then by the conclusion of the theorem, $z$, the generator of $\mathfrak{Z}_{n}$, is in $\mathfrak{Z}_{n+d-1}$ but not $\mathfrak{Z}_{n+d}$. So $z$ commutes with generators $v_{0}$ through $v_{n+d-2}$ and anticommutes with $v_{n+d-1}$. Setting $u$ as above and $\beta=A d(u) \circ \alpha$ we see that $u_{0}$ through $u_{n+d-2}$ coincide with $v_{0}$ through $v_{n+d-2}$, but $u_{n+d-1}=\beta\left(u_{n+d-2}\right)=\beta\left(v_{n+d-2}\right)=$ $u^{*} v_{n+d-1} u=\lambda_{0} z v_{n+d-1}, u_{n+d}=u^{*} \alpha\left(\lambda_{0} z v_{n+d-1}\right) u=\lambda_{1} z^{r_{0}} \alpha(z) v_{n+d}, u_{n+d+1}=$ $\lambda_{2} z^{r_{1}} \alpha(z)^{r_{0}} \alpha^{2}(z) v_{n+d+1}$, and so on, for scalars $\lambda_{i}$ of modulus 1 .

As $\alpha^{d-1}(z)$ is in $\mathfrak{Z}_{n+2 d-2}$, and therefore commutes with $v_{0}$ through $v_{n+2 d-3}$, it follows that $\alpha^{d}(z)$ commutes with $v_{1}$ through $v_{n+2 d-2}$. As $\mathfrak{Z}_{n+2 d-1}$ is trivial, however, and as $\mathfrak{A}_{n+2 d-1}$ is generated by $v_{0}$ through $v_{n+2 d-2}$ we conclude that $\alpha^{d}(z)$ anticommutes with $v_{0}$. Therefore the following statements are true:

$u_{0}, u_{1}, \ldots, u_{n+d-2}$ agree with $v_{0}, v_{1}, \ldots, v_{n+d-2}$, respectively,

$u_{n+d-1}$ is a scalar multiple of $z v_{n+d-1}$

$u_{n+d}$ is a scalar multiple of $z^{s_{0}} \alpha(z) v_{n+d}$, for some exponent $s_{0}$,

$u_{n+d+1}$ is a scalar multiple of $z^{s_{1}} \alpha\left(z^{s_{0}}\right) \alpha^{2}(z) v_{n+d+1}$, for some exponent $s_{1}$,

$u_{n+2 d-2}$ is a scalar multiple of $z^{s_{d-2}} \cdots \alpha^{d-2}(z)^{s_{0}} \alpha^{d-1}(z) v_{n+2 d-2}$, and

$u_{n+2 d-1}$ is a scalar multiple of $z^{s_{d-1}} \cdots \alpha^{d-1}(z)^{s_{0}} \alpha^{d}(z) v_{n+2 d-1}$.

It follows that the bitstream entries $b_{0}$ through $b_{n+2 d-2}$ coincide with $a_{0}$ through $a_{n+2 d-1}$ but, as $u_{n+2 d-1}$ is a scalar multiple of $z^{s_{d-1}} \cdots \alpha^{d-2}\left(z^{s_{1}}\right) \alpha^{d-1}\left(z^{s_{0}}\right) \alpha^{d}(z) v_{n+2 d-1}$, and $u_{0}$ anticommutes with $\alpha^{d}(z)$, it follows that $b_{n+2 d-1} \neq a_{n+2 d-1}$.

An argument similar to the one given in the original case shows that $\mathfrak{A}_{m}=\mathfrak{B}_{m}$ for all $m$, so $\beta$ is a binary shift on $R$ cocycle conjugate, but not conjugate, to $\alpha$. So we have nearly completed the proof of the following.

Theorem 5. Any binary shift on the hyperfinite $I I_{1}$ factor $R$ is cocycle conjugate to at least countably many others.

End of proof. Let $\beta_{1}$ be the binary shift $\beta$ constructed above. To construct $\beta_{2}$ from $\alpha$, choose $n_{2} \in \mathbb{N}$ such that $n_{2}>n_{1}=n$ and $\nu_{n_{2}-1}=0, \nu_{n_{2}}=1$, and mimic the construction already made for $\beta$. Note that the first place where the bitstreams 
for $\beta_{2}$ and $\alpha$ differ will occur past the first place where the bitstream for $\beta_{1}$ and $\alpha$ differ, so $\beta_{1}$ and $\beta_{2}$ are not conjugate. Continuing this process we can construct countably many binary shifts which are mutually non-conjugate but which are all cocycle conjugate to $\alpha$.

Definition 2. For $k \in \mathbb{N}$, a binary shift $\alpha$ on $R$ is said to have commutant index $k$ if the relative commutant algebra $\alpha^{k}(R)^{\prime} \cap R$ is non-trivial and $k$ is the first non-negative integer for which this is the case. If $\alpha^{k}(R)^{\prime} \cap R=\mathbb{C} I$ for all $k \in \mathbb{N}$ then we say that $\alpha$ has infinite commutant index.

Here are a few facts about the commutant index of a binary shift. The minimal commutant index is $2,[\mathrm{~J}]$, and for every $k \in\{\infty, 2,3 \ldots\}$ there are binary shifts of commutant index $k,[\operatorname{Pr} 01$, Theorem 5.5]. A binary shift has finite commutant index if and only if its bitstream is eventually periodic, [BY, Theorem 5.8]. The commutant index is a cocycle conjugacy invariant [BY], [Pr98, Theorem 5.5].

Remark 2. By [Pr 98a, Corollary 4.10], all binary shifts of commutant index 2 are cocycle conjugate, so it makes sense to ask whether, given a binary shift $\alpha$ of index 2 , the list $\left\{\alpha, \beta_{k}, k \in \mathbb{N}\right\}$ includes all binary shifts of commutant index 2. To see that this is not the case, note from the construction of the $\beta_{k}$ 's in the proof of the theorem that for every $m \in \mathbb{N}$ the algebra generated by the first $m$ generators for $\alpha$ coincides with the algebra generated by the first $m$ generators for $\beta_{k}$. Hence they have the same centers and therefore, by Theorem 3 , the same nullity sequence. Suppose $n \in \mathbb{N}$ is the first positive integer for which $\nu_{n}=\operatorname{null}\left(\mathcal{A}_{n}\right)=0$. Set $c_{j}=0$ for $0 \leq j \leq n-1$ and $c_{n}=1$. Then by [Pr98] $c_{0}, \ldots, c_{n}$ may be completed to form a bitstream which corresponds to a binary shift $\gamma$ of commutant index 2. Note, however, that the corresponding Toeplitz matrix $\mathcal{C}_{n}$ is the zero matrix, with nullity $n$. Since $\operatorname{null}\left(C_{n}\right) \neq \operatorname{null}\left(A_{n}\right)=0, \gamma$ is not in the list $\alpha, \beta_{k}, k \in \mathbb{N}$.

Remark 3. In [Pr98] it was shown that if a binary shift $\alpha$ of finite commutant index has a nullity sequence which agrees except at finitely many places with the nullity sequence of $\alpha_{i n f t y}$, then $\alpha$ and $\alpha_{\infty}$ are cocycle conjugate.

\section{Problems.}

(1) A binary shift $\alpha$ is said to be of infinite commutant index if $\alpha^{k}(R)^{\prime} \cap R=\mathbb{C} I$ for all $k$. Is there an $\alpha$ 's of infinite commutant index which is cocycle conjugate to uncountably many binary shifts?

(2) Are any two binary shifts of infinite commutant index cocycle conjugate?

\section{REFERENCES}

[AP] W. B. Arveson and G. Price, The structure of spin systems, Internat. J. Math. 14 (2003), 119-137. 
[BY] D. Bures and H. S. Yin, Outer conjugacy of shifts on the hyperfinite II $I_{1}$ factor, Pacific J. Math. 142 (1990), 245-257.

$[\mathrm{J}] \quad$ V. F. R. Jones, Index for subfactors, Invent. Math., 1-25.

[KR] R. V. Kadison and J. R. Ringrose, Fundamentals of the theory of operator algebras, Volume II, Academic Press, 1986.

[Po] R. T. Powers, An index theory for semigroups of $*$-endomorphisms of $\mathcal{B}(\mathcal{H})$ and type $I I_{1}$ factors, Can. J. Math 40 (1988), 86-114.

[PP] R. T. Powers and G. Price, Cocycle conjugacy classes of shifts on the hyperfinite $I I_{1}$ factor, J. Funct. Anal. 121 (1994), 275-295.

[Pr87] G. Price, Shifts on type II $I_{1}$ factors, Can. J. Math. 39 (1987), 492-511.

[Pr98a] G. Price, Cocycle conjugacy classes of shifts on the hyperfinite II 1 factor. II., J. Operator Theory 39 (1998), 177-195.

[Pr98] G. Price, Shifts on the hyperfinite II 1 factor, J. Func. Anal. 156 (1998), 121-169.

[Pr99] G. Price, On the classification of binary shifts of finite commutant index, Proc. National Acad. Sci. 96 (1999), 14700-14705.

Department of Mathematics, United States Naval Academy, Annapolis MD 21402, U.S.A.

E-mail address: glp@usna.edu 